\title{
BMJ Open A randomised placebo-controlled double-blind multicentre trial comparing antibiotic therapy with placebo in the treatment of uncomplicated acute appendicitis: APPAC III trial study protocol
}

Suvi Sippola, ${ }^{1,2}$ Juha Grönroos, ${ }^{1,2}$ Ville Sallinen, ${ }^{3}$ Tero Rautio, ${ }^{4}$ Pia Nordström, ${ }^{5}$ Tuomo Rantanen, ${ }^{6}$ Saija Hurme, ${ }^{7}$ Ari Leppäniemi, ${ }^{3}$ Sanna Meriläinen, ${ }^{4}$ Johanna Laukkarinen, ${ }^{5}$ Heini Savolainen, ${ }^{6}$ Johanna Virtanen, ${ }^{8}$ Paulina Salminen ${ }^{1,2}$

To cite: Sippola S, Grönroos J, Sallinen $\mathrm{V}$, et al. A randomised placebo-controlled doubleblind multicentre trial comparing antibiotic therapy with placebo in the treatment of uncomplicated acute appendicitis: APPAC III trial study protocol. BMJ Open 2018;8:e23623. doi:10.1136/ bmjopen-2018-023623

- Prepublication history for this paper is available online. To view these files, please visit the journal online (http://dx.doi org/10.1136/bmjopen-2018023623).

Received 15 April 2018 Revised 19 August 2018 Accepted 4 September 2018

Check for updates

(C) Author(s) (or their employer(s)) 2018. Re-use permitted under CC BY-NC. No commercial re-use. See rights and permissions. Published by BMJ.

For numbered affiliations see end of article.

Correspondence to Dr Paulina Salminen; paulina.salminen@tyks.fi

\section{ABSTRACT}

Introduction Recent studies show that antibiotic therapy is safe and feasible for CT-confirmed uncomplicated acute appendicitis. Spontaneous resolution of acute appendicitis has already been observed over a hundred years ago. In CT-confirmed uncomplicated acute diverticulitis (left-sided appendicitis), studies have shown no benefit from antibiotics compared with symptomatic treatment, but this shift from antibiotics to symptomatic treatment has not yet been widely implemented in clinical practice. Recently, symptomatic treatment of uncomplicated acute appendicitis has been demonstrated in a Korean open-label study. However, a double-blinded placebo-controlled study to illustrate the role of antibiotics and spontaneous resolution of uncomplicated acute appendicitis is still lacking.

Methods and analysis The APPAC III (APPendicitis ACuta III) trial is a multicentre, double-blind, placebo-controlled, superiority randomised study comparing antibiotic therapy with placebo in the treatment CT scan-confirmed uncomplicated acute appendicitis aiming to evaluate the role of antibiotics in the resolution of uncomplicated acute appendicitis. Adult patients (18-60 years) with CT scanconfirmed uncomplicated acute appendicitis (the absence of appendicolith, abscess, perforation and tumour) will be enrolled in five Finnish university hospitals. Primary endpoint is success of the randomised treatment, defined as resolution of acute appendicitis resulting in discharge from the hospital without surgical intervention within 10 days after initiating randomised treatment (treatment efficacy). Secondary endpoints include postintervention complications, recurrent symptoms after treatment up to 1 year, late recurrence of acute appendicitis after 1 year, duration of hospital stay, sick leave, treatment costs and quality of life. A decrease of 15 percentage points in success rate is considered clinically important difference. The superiority of antibiotic treatment compared with placebo will be analysed using Fisher's one-sided test and $\mathrm{Cl}$ will be calculated for proportion difference.

Ethics and dissemination This protocol has been approved by the Ethics Committee of Turku University Hospital and
Strengths and limitations of this study

To our knowledge, APPAC III trial (APPendicitis ACuta III) is the first double-blinded placebo-controlled trial comparing antibiotic therapy with placebo to evaluate the role of antibiotic therapy in the treatment of uncomplicated acute appendicitis and also assess possible spontaneous resolution of uncomplicated acute appendicitis.

- The double-blinding with placebo as the other treatment arm helps both to minimise bias associated with outcome evaluation and to further evaluate possible symptomatic care of uncomplicated acute appendicitis.

- Multicentre original study with thorough recording of data on all evaluated patients in order to help understand possible selection bias.

- Implementing abdominal contrast-enhanced CT imaging with a radiation dose optimised protocol followed by a radiological diagnosis prior to enrolment in all adult patients with suspected acute appendicitis.

- Based on the combination of required hospital pharmacy resources enabling accurate treatment blinding, the emergency surgery setting and the senior surgeon recruitment, the time frame for patient enrolment is restricted to main office hours anticipated to limit and slow down the patient enrolment.

the Finnish Medicines Agency (FIMEA). The findings will be disseminated in peer-reviewed academic journals.

Trial registration number NCT03234296; Pre-results.

\section{INTRODUCTION}

Appendectomy has been the standard treatment for acute appendicitis for over a century. ${ }^{1}$ More than 300000 appendectomies 
are performed annually in the USA. ${ }^{2}$ Although appendectomy is generally well tolerated, it is a major surgical intervention and can be associated with postoperative morbidity. ${ }^{3-5}$

Since Fitz $^{6}$ described the relationship between the appendix and pelvic abscess and McBurney ${ }^{7}$ demonstrated reduced morbidity from pelvic infections attributable to appendectomy, it has been thought that acute appendicitis invariably progresses to perforation. Emergency appendectomy was first advocated because of the high mortality related to perforated appendicitis, especially in the preantibiotic era, combined with the assumption of the natural course of acute appendicitis always evolving to perforated disease. However, already in 1886 Fitz reported that one-third of patients in a large autopsy series from the pre-appendectomy era had evidence of prior appendiceal inflammation suggesting spontaneous resolution of acute appendicitis. ${ }^{6}$ Large epidemiological studies have shown that complicated or perforated and uncomplicated or non-perforated appendicitis have followed different epidemiological trends suggesting different pathophysiology for the two forms. ${ }^{2}$

In recent years, the treatment of acute appendicitis has been under active debate and discourse as increasing amount of evidence has shown that the majority of patients with uncomplicated acute appendicitis may be treated with antibiotics alone instead of surgery. ${ }^{8-14}$ The original APPAC (APPendicitis ACuta) trial reported that $73 \%$ of patients with CT-confirmed uncomplicated acute appendicitis did not receive appendectomy during a 1-year follow-up period, and those patients who had appendectomy did not experience major complications. ${ }^{8}$ These results suggested that CT-proven uncomplicated acute appendicitis is not a surgical emergency and antibiotic therapy is a safe first-line treatment of uncomplicated acute appendicitis, which has since been endorsed in recent meta-analyses. ${ }^{14-16}$

Acute appendicitis is clinically very similar to acute diverticulitis (left-sided appendicitis), and this resemblance has been shown also in epidemiological studies suggesting a common underlying pathogenesis. ${ }^{2}$ The treatment of CT scan-confirmed uncomplicated acute diverticulitis has experienced a shift from antibiotic treatment towards spontaneous resolution. There is one randomised trial, ${ }^{1718}$ one population-based study, ${ }^{19}$ one case-control study ${ }^{20}$ and two prospective observational studies ${ }^{21} 22$ that have shown no benefit of antibiotic therapy in uncomplicated acute diverticulitis. The reported complication rates in these studies are low (approximately 2\%) and even outpatient management without antibiotics in uncomplicated acute diverticulitis has been shown to be feasible, well functioning and safe. ${ }^{21} 22$ Similarly, uncomplicated acute appendicitis may not require surgical intervention, and it might even experience spontaneous resolution. ${ }^{2}$ Recently, Park et $a l^{23}$ reported promising results regarding possible spontaneous resolution in uncomplicated acute appendicitis, but their study was single-blind (participants only), not double-blind. A double-blinded design is imperative in studying the possible spontaneous resolution of uncomplicated acute appendicitis to minimise bias by both reducing the potential for a treatment effect in patients and the risk of the researchers reporting greater effects in the treatment group or lesser effects in the placebo control group.

The diagnosis of acute appendicitis remains challenging even though it is the most common reason for surgical emergency room visits. Traditionally, the diagnosis has been based on patient history, clinical suspicion and laboratory findings, but the accuracy of clinical diagnosis in acute appendicitis without preoperative imaging is not optimal for combined patient groups of men and women. ${ }^{24}$ Further, clinical findings or laboratory markers do not provide a reliable enough method to establish the clinically relevant differential diagnosis between uncomplicated and complicated acute appendicitis without CT imaging. ${ }^{25}$

With promising results of conservative treatment of uncomplicated acute appendicitis with antibiotics, the correct differential diagnosis between the two different forms of acute appendicitis is crucial. ${ }^{815}$ CT imaging has become the gold standard for appendicitis imaging due to its superiority in diagnosing acute appendicitis and currently also its ability to differentiate between uncomplicated and complicated acute appendicitis. ${ }^{26} 27$ The advantages of CT imaging are high accuracy, availability, ease of performance and interpretation, and that it is rarely affected by bowel gas, severe abdominal pain or extreme body habitus. ${ }^{28} 29$ The increased use of preoperative CT imaging is also cost-efficient through increased diagnostic accuracy and the avoidance of unnecessary appendectomies. ${ }^{30-32}$ The inevitable disadvantage of CT imaging is exposure to ionising radiation and as the majority of patients with acute appendicitis are young adults, the imminent need to decrease radiation dose has been approached through developing low-dose CT protocols. ${ }^{33}$ Despite a recent meta-analysis ${ }^{34}$ showing equal accuracy of low-dose and standard CT in diagnosing acute appendicitis, low-dose CT protocols have yet to be thoroughly implemented in clinical practice.

Our study group conducted a prospective interpatient randomised observational study (the OPTICAP trial, NCT02533869), in which the same patient with suspected acute appendicitis underwent protocol sequence randomised consecutive imaging with both optimised standard and low-dose CT protocols with intravenous contrast media. The primary objective of the OPTICAP trial was to optimise and evaluate the feasibility of a low-dose CT protocol in diagnosing acute appendicitis in the clinical phase of the study; the protocols were first evaluated in the phantom model in 'in vitro' phase of the OPTICAP trial, ${ }^{35}$ based on which a body mass index (BMI) of $<30 \mathrm{~kg} / \mathrm{m}^{2}$ was set as a limit for low-dose CT imaging as we demonstrated additional noise to the CT caused by the adipose tissue in BMI exceeding $30 \mathrm{~kg} / \mathrm{m}^{2}$ potentially affecting the readability of the images. The 
most optimal low-dose and standard CT imaging protocols based on the OPTICAP trial were implemented in all APPAC III trial hospitals to be used for the enrolment in the current APPAC III trial and the diagnostic accuracy of contrast enhanced low-dose CT was not inferior to standard CT in diagnosing acute appendicitis or distinguishing between uncomplicated and complicated acute appendicitis in patients with high likelihood of acute appendicitis. Low-dose CT also enabled significantly reduced radiation dose. ${ }^{36}$

To our knowledge, so far there are no double-blinded randomised controlled trials (RCTs) comparing placebo with antibiotic therapy in the treatment of uncomplicated acute appendicitis. The APPAC III trial aims to assess the role of antibiotic therapy in the resolution of CT-confirmed uncomplicated acute appendicitis and the spontaneous resolution of uncomplicated acute appendicitis. In the APPAC III trial, we aim to evaluate intravenous followed by per oral antibiotic therapy versus intravenous followed by per oral placebo in terms of treatment efficacy, postintervention complications, length of hospital stay, treatment costs and appendicitis recurrence.

\section{METHODS AND DESIGN Study design}

The APPAC III trial is a randomised double-blinded, placebo-controlled, superiority multicentre trial comparing antibiotic therapy with placebo in the treatment of CT-confirmed uncomplicated acute appendicitis. The protocol was drafted in accordance with the SPIRIT (Standard Protocol Items: recommendations for Interventional Trials) statement. ${ }^{37}$ The trial has been registered at ClinicalTrials.gov prior to study patient enrolment. All patients participating in the study will give written consent obtained by the researchers. The study flow is illustrated in figure 1.

The aim of the study is to compare antibiotic therapy with placebo in the treatment of uncomplicated acute appendicitis to evaluate the role of antibiotic therapy in appendicitis resolution. The study hypothesis is that antibiotic therapy is necessary in the treatment of uncomplicated acute appendicitis and that antibiotic therapy is superior to supportive care without antibiotics with the primary endpoint evaluated at 10 days after the intervention.

\section{Patient selection and diagnostic procedures}

Eligible for inclusion are adult patients 18-60 years old of both sexes admitted to the emergency department of one of the participating hospitals with suspected acute appendicitis in whom a CT-confirmed uncomplicated acute appendicitis is diagnosed. All adult patients with a clinical suspicion of acute appendicitis will be studied carefully by attending surgeons at the emergency departments of the participating hospitals. Clinical history, physical investigation and laboratory tests (blood haemoglobin, white cell count, $\mathrm{C}$ reactive protein (CRP), creatinine, urine analysis and in female patients also human chorionic gonadotropin) are evaluated. Prior to pain medication administration, pain scores using Visual Analogue Scale (VAS) will be recorded. If clinical history and physical examination suggest acute appendicitis, the patient will undergo CT imaging with either an optimised low-dose $100 \mathrm{kV}^{35}$ $\left(\mathrm{BMI}<30 \mathrm{~kg} / \mathrm{m}^{2}\right)$ or a standard $120 \mathrm{kV}\left(\right.$ BMI $\left.>30 \mathrm{~kg} / \mathrm{m}^{2}\right)$ contrast-enhanced CT. Alternatively, a corresponding low-dose protocol with automated tube voltage $(\mathrm{kV})$ selection is used in all sized patients if the technique is available in the study hospitals.

\section{Inclusion criteria}

The inclusion criteria are: (1) age 18-60 years, (2) diagnosis of uncomplicated acute appendicitis confirmed by CT scan defined by the following criteria: appendiceal diameter exceeding $6 \mathrm{~mm}$ with thickened and enhancing wall and periappendiceal oedema and/orminor fluid collection, and the absence of the criteria of complicated appendicitis (see Exclusion criteria section). The CT findings will be evaluated using a standardised CT scan report sheet (box 1).

\section{Exclusion criteria}

The exclusion criteria are the following: (1) age under 18 or over 60 years, (2) pregnancy or lactation, (3) allergy to contrast media or iodine, (4) allergy or contraindication to antibiotic therapy, (5) administration of antibiotics prior to enrolment, (6) renal insufficiency or serum creatinine value exceeding the upper reference limit, (7) type 2 diabetes mellitus and metformin medication, (8) severe systemic illness (eg, malignancy, severe sepsis, medical condition requiring immunosuppressant medication), (9) inability to cooperate and give informed consent and (10) complicated acute appendicitis in the CT scan. A radiological diagnosis of complicated acute appendicitis is defined as typical findings of appendicitis with at least one of the following: appendicolith, periappendiceal abscess, perforation (free air or finding of diffuse peritonitis) or suspicion of an appendiceal tumour (box 1). We have reported high inter-rater agreement of differential diagnosis between uncomplicated and complicated acute appendicitis using these criteria in our OPTICAP trial. Contraindications for the use of antibiotics include either allergy to the antibiotic regimen, auxiliary substance of the drug or interaction with other medications of the patient. In the case of quinolones, epilepsy and previously diagnosed tendinitis or tendon rupture related to quinolone treatment are contraindications. Other overall contraindications to antibiotic treatment in general including pregnancy or lactation and age under 18 years do not apply as these patients will not be evaluated for study enrolment based on general exclusion criteria.

\section{Excluded patients}

In order to both understand any selection bias and to enable a thorough recording of the patient population with suspected acute appendicitis, all patients undergoing 


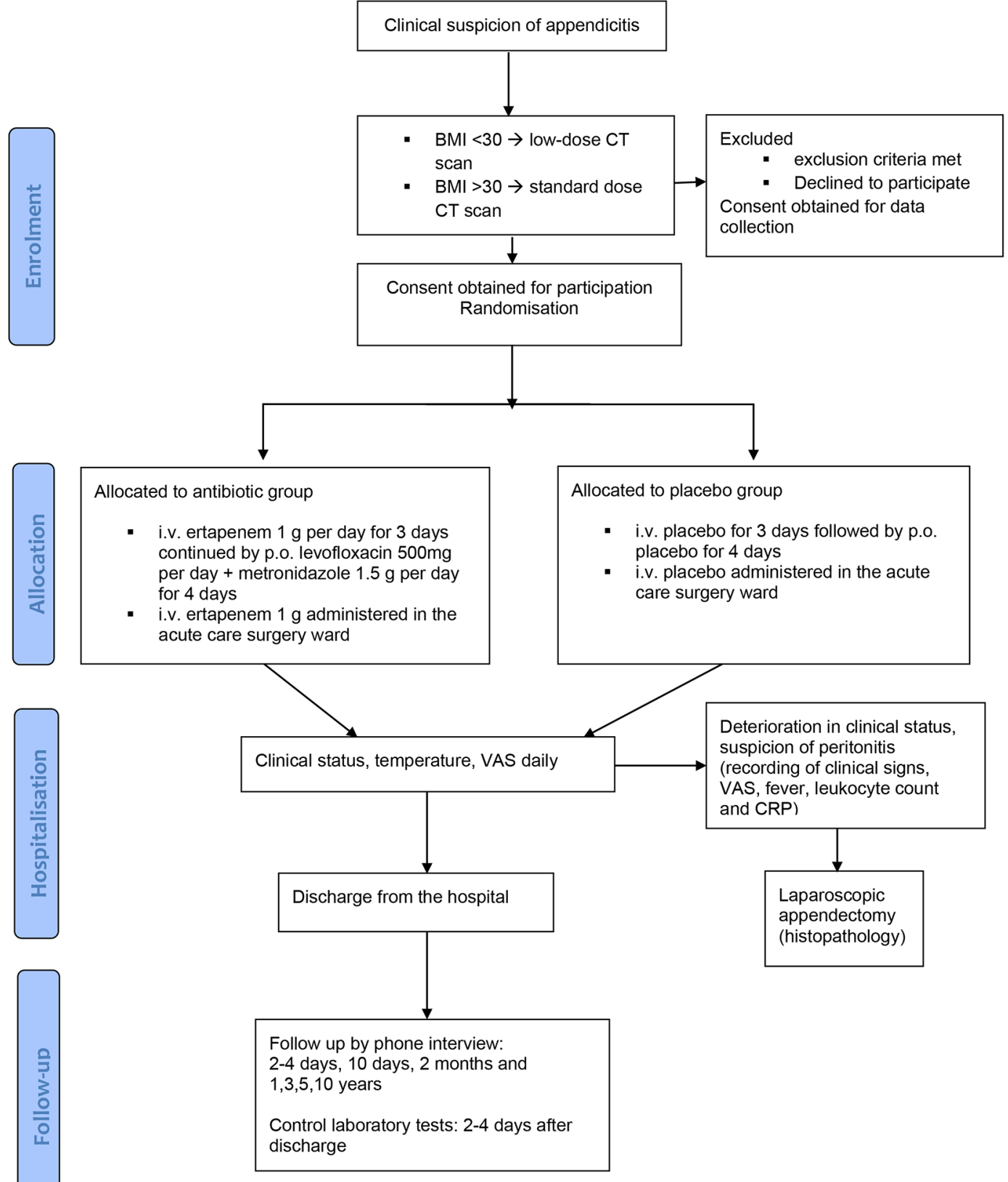

Figure 1 Flow chart of the APPAC III (APPendicitis ACuta III) study protocol. BMI, body mass index; CRP, C reactive protein; i.v., intravenous; p.o., orally; VAS, Visual Analogue Scale.

a CT for suspected acute appendicitis will be recorded in the study online database containing information about their demographics, symptoms, clinical findings, laboratory values and CT findings. All patients will be informed about data collection and they will sign an informed consent for this data collection. Clinical history, physical investigation, VAS pain score and laboratory tests will be recorded in the study online database.

\section{Randomisation}

Patients are randomised with a 1:1 equal allocation ratio to antibiotic therapy or placebo. Randomisation will be made by a safety statistician of the trial by centre using random permuted blocks. The randomisation and the used randomisation blocks will be blinded to the investigators. The randomisation listing will be made available only to the safety statistician and the hospital pharmacies based on patient numbers. After written informed consent, randomisation of the patient will be performed using the online database by the senior research surgeons in each participating hospital. The pharmacists will conceal the allocated treatment by labelling the study drugs in exactly similar containers identifiable only by the patient identification numbers. The randomised treatment will be initiated in the acute care surgery ward. To ensure patient safety regarding the possible emergency opening of the double-blinded code, sealed randomisation lists for each 
Box 1 Structured reporting template of abdominal CT: APPAC (APPendicitis ACuta) multicentre

I. Descriptive part of the report: technique and findings in the whole abdomen

II. Structured report of appendix:

1. Appendix visualisation Report one of the following:

- Not visualised/partly or unclearly visualised/completely visualised

2. Appendix transverse diameter $(\mathrm{mm})$ :

3. Probability of appendicitis Report one of the following:

- Not likely/rather unlikely/rather likely/very likely

4. Categorisation of the appendicitis Report either I or II, if any:

I. Uncomplicated appendicitis: transverse diameter $>6 \mathrm{~mm}$ with typical findings

Wall thickening and enhancement

Periappendiceal oedema and/or minor amount of fluid

II. Complicated appendicitis: Appendicitis with at least one of the following:

- Appendicolith: $>3 \mathrm{~mm}$ stone within appendix

- Abscess: periappendiceal walled of collection with enhancing walls

- Perforation: appendiceal wall enhancement defect and periappendiceal excess of fluid and/or infectious phlegmon and/or extraluminal air

- Tumour: tumour-like prominence of appendix

5. Other diagnosis: Report if any

- Diverticulitis/complicated ovarian cyst/pelvic inflammatory disease/colitis/lleitis/intestinal obstruction or ileus/ureter stone/hydronephrosis/tumour/other diagnosis

hospital are available at each hospital and are opened only in cases of emergency, that is, suspected allergic reaction to treatment intervention.

The allocated randomisation time frame for the APPAC III trial varies slightly between the hospitals due to local differences in the hospital research pharmacy resources, but in general the randomisation is possible during regular working hours from Monday to Thursday. We have another concurrently ongoing national openlabel randomised trial (APPAC II) comparing per oral monotherapy versus combination of intravenous antibiotic followed by per oral antibiotic therapy aiming to optimise the antibiotic treatment of CT scan-confirmed uncomplicated acute appendicitis. This APPAC II trial is also run by our study group and outside hospital pharmacy hours, patients are recruited to participate in the APPAC II trial. During study hospital pharmacy working hours all eligible patients are informed of both ongoing trials, but they are first asked to participate in the APPAC III trial. In case they decline to participate in the APPAC III trial, they also have the possibility of participating in the APPAC II trial in four out of the five APPAC III study hospitals (Helsinki University Hospital is not taking part in the APPAC II trial). The trial selection is based only on the hospital working hours and thus possible selection bias caused by the concurrent trials is highly unlikely.

\section{Blinding}

After enrolment, the patient is randomised by the research surgeon using the online database and receives a randomisation number. The research surgeon informs the hospital pharmacy of this patient number by phone initiating the drug manufacturing using the randomisation list available only at the hospital pharmacy. The manufacturing of the drugs starts with the first intravenous dose and at the same time the manufacturing of the oral antibiotics or placebo is initiated. The hospital pharmacy manufactures the antibiotic and placebo treatments to appear exactly identical. The intravenous solutions in similar intravenous bags and labels are delivered to the surgical ward, where they are administered to the patient according to the predefined administration schedule and the administration time (ie, door-to-needle time) is recorded. The intravenous antibiotic is ertapenem sodium $1 \mathrm{~g}$ per dose and the placebo consists of $0.9 \%$ saline solution. The oral capsules have similar identical appearance and labelling using only the patient randomisation number.

The coloured capsules consist of gelatine and are identical in appearance, and do not have specific smell or taste also eliminating the distinct metallic taste of metronidazole. Medicinal substances are ground and the powder is placed into the capsules. The coloured capsules were chosen due to levofloxacin tablets having red spots and using a coloured capsule eliminates the possibility of detecting differences of the powdered substances through the capsules. Hospital pharmacy manufactures a total of four levofloxacin capsules using four tablets of $500 \mathrm{mg}$ levofloxacin and $1.22 \mathrm{~g}$ microcrystal cellulose, and four corresponding placebo capsules using $3.14 \mathrm{~g}$ of microcrystal cellulose. Twelve metronidazole capsules are manufactured from $12500 \mathrm{mg}$ metronidazole tablets and $3.22 \mathrm{~g}$ of microcrystal cellulose. Corresponding number of placebo capsules are manufactured from $8.16 \mathrm{~g}$ of microcrystal cellulose. Patients receive two similar plastic medicine bottles with labels and dose directions of administration on discharge; one bottle contains levofloxacin or placebo and the other metronidazole or placebo. The study drugs will be administered by nurses at the acute care surgery ward and they will not be handled by the study physicians, who assess the eligibility of the patients, obtain consent, enrol patients, care for patients during the study, collect data and assess outcomes.

In order to ensure the hospital pharmacy drug manufacturing and adequate blinding in real-life clinical practice, we conducted a pilot study between May 2017 and July 2017 enrolling five pilot patients at two research hospital (Turku and Kuopio). No changes were made to the study protocol interventions or the hospital pharmacy arrangements based on this pilot study. After completion of the pilot study ensuring the feasibility of the drug manufacturing process and study flow in July 2017, the actual APPAC III trial enrolment was initiated in September 2017. 


\section{Sample size calculation}

Sample size calculations were based on one-sided Pearson's $\chi^{2}$ test for two proportions. Sample size was calculated from an estimated success rate of $94 \%$ during the hospitalisation in antibiotic group. ${ }^{8}$ A power of 0.8 (1ß) and one-sided significance level $(\alpha)$ of 0.05 were used in calculations. Based on the pilot study enrolment speed and the previously recognised challenges in conducting the trial in real-life emergency setting, we need to take into account the anticipated enrolment delays to assure the completion of this trial within reasonable time. These challenges consisting of emergency surgery patient enrolment dependent on hospital research pharmacy services available only during standard working hours, the requirement for senior surgeon enrolment and the discrepancy between the admission hours of patients with appendicitis and hospital pharmacy working hours, mandated us to create three different scenarios for study power analysis and the number of patients to be enrolled.

In scenario $\mathrm{A}$, a decrease of 15 percentage points in success rate is considered clinically important difference leading to estimated $79 \%$ success rate in placebo group. This 15-percentage point difference in rescue appendectomy rate was determined arbitrarily based on clinical relevance as at the time of study planning and initiation, no information about the symptomatic treatment success of uncomplicated acute appendicitis was available. We calculated that to detect a 15-percentage points difference (antibiotics-placebo) between groups, 64 patients per group is needed. With an estimated dropout rate of $10 \%$ total of 142 patients, 71 patients per group will be enrolled in the study. In scenario B, clinically important difference is 20 percentage points, estimated success rate in placebo group is $74 \%$ and to detect this difference 41 patients per group is needed. With an estimated dropout rate of $10 \%$ total of 92 patients, 46 patients per group will be enrolled in the study. In scenario C, clinically important difference is 25 percentage points, estimated success rate in placebo group is $69 \%$ and to detect this difference 29 patients per group is needed. With an estimated dropout rate of $10 \%$ total of 64 patients, 32 patients per group will be enrolled in the study. Targeted minimum sample size per study hospital will be 10 patients. One-sided test will be used as our hypothesis is that antibiotic treatment is more effective treatment than placebo. Sample size calculations were performed using Power procedure in SAS System for Windows, V.9.4.

On 1 June 2019, a study committee consisting of the outside safety monitoring committee and the core research group will assess which one of these scenarios will be chosen based on patient enrolment with plan A being the target scenario. Active recruitment at this point will be continued until a decision is made on the clinically realistic enrolment scenario. The date of evaluation is set approximately 1 year after the last study hospital has initiated enrolment. If the patient enrolment on 1 June 2019 has reached the patient number in scenario B (92 patients), the recruitment will continue until original target scenario A (142 patients) is reached. If scenario B enrolment target has not been reached at this evaluation time point, but scenario $\mathrm{C}$ patient number (64 patients) has been recruited, the scenario B with 92 patients will be the new target scenario. If the patient enrolment has not reached scenario $\mathrm{C}$ on 1 June 2019, the new target scenario will be $\mathrm{C}$ with 64 patients.

\section{Study setting and feasibility}

Eligible patients are recruited from all five Finnish university hospitals (Turku, Oulu, Helsinki, Tampere and Kuopio). Trial participation is restricted to the university hospitals based on essential hospital pharmacy resources. The pilot phase of the study began at the Turku and Kuopio University Hospitals in April 2017, with the study officially commencing actual patient enrolment at these two hospitals in September 2017. Required hospital pharmacy arrangements were finalised at all study hospitals in March 2018 with initiation of patient enrolment in all five hospitals during April 2018.

\section{Interventions}

To ensure patient safety in the placebo group, all patients will be monitored in the hospital for the duration of the intravenous treatment, that is, for three intravenous infusions (approximately 3 days).

\section{Antibiotic therapy group}

Antibiotic regimen is identical to the previously published APPAC trial as the study needed antibiotic therapy with proven efficacy in order to compare an efficient therapy with placebo. APPAC III trial patients randomised to the antibiotic therapy group will receive intravenous ertapenem sodium $1 \mathrm{~g}$ administered for 3 days with the first dose given in the acute care surgery ward. Intravenous ertapenem will be followed by per oral levofloxacin $500 \mathrm{mg}$ administered once daily and per oral metronidazole $500 \mathrm{mg}$ administered three times per day for 4 days resulting in a total treatment duration of 1 week. Antibiotic therapy has been shortened from the original APPAC trial, in which the antibiotic treatment duration was 10 days with 1 week of per oral antibiotics.

\section{Placebo group}

For patients randomised to the placebo group, intravenous and per oral placebo will be administered according to an exactly identical protocol with the antibiotic therapy group.

\section{Follow-up during hospitalisation}

During hospitalisation the following parameters will be recorded every 24 hours: VAS or changes in VAS, leucocyte count, CRP, temperature and clinical findings at patient reassessment. The researcher surgeon will reassess the patient twice daily and this will allow for us to evaluate the clinical response on a daily basis. Pain medication is prescribed according to standard hospital protocol. Pain management is multimodal including parenteral or oral NSAID and paracetamol with opiates being administered, 
but only if necessary and no routine pain management is scheduled. On admittance to the ward, patients will initially be kept from eating and drinking until the first status control conducted at 12-24 hours after admittance to the hospital. If the clinical condition is the same or improved, patients are allowed to eat and drink. In case of worsened clinical condition, the patients will be kept from eating and drinking until the next reassessment. If the patient is suspected of not responding to the administered therapy based on clinical deterioration signs combined with laboratory findings (signs of peritonitis, persisting fever, increasing level of pain, rising white cell count or CRP), the patient will be operated based on the surgeon's decision and the reasons for proceeding to laparoscopic appendectomy will be recorded in the database. The approach of direct appendectomy in cases of suspected non-responsiveness to randomised treatment was chosen to ensure patient safety as the results of symptomatic treatment of uncomplicated acute appendicitis are scarce and there are no previous double-blind trials addressing symptomatic treatment. To minimise bias associated to the double-blinded study setting with the possibility of placebo treatment potentially lowering the threshold of appendectomy within the surgeons on call, the patients are evaluated mainly by the study senior surgeons with both vast clinical experience and thorough knowledge of the previous APPAC trial and the current APPAC III trial protocol. These research surgeons monitor the patient and make the decision to convert to operative treatment if necessary; they must record their rationale for this decision in the database. The operative findings and the histopathology of the appendix will be also recorded in the database.

After the initial hospitalisation, recurrent acute appendicitis will be diagnosed on a clinical basis and all patients with a clinically evident suspicion of appendicitis recurrence will undergo a laparoscopic appendectomy without further imaging. Patients with atypical or vague symptoms undergo regular clinical evaluation including possible imaging based on the assessment of the surgeon on call. The operative findings and the histopathology of the appendix will be recorded in the database.

\section{OUTCOME PARAMETERS AND STATISTICAL ANALYSIS Primary outcome}

The primary endpoint of the APPAC III trial is the success of the randomised treatment (treatment efficacy). Treatment success is defined as the resolution of acute appendicitis with study treatment resulting in discharge from the hospital without receiving appendectomy and treatment efficacy will be evaluated at 10 days after initiation of the randomised treatment.

\section{Secondary outcomes}

Secondary endpoints include postintervention complications (possible postoperative complications classified according to the Clavien-Dindo classification ${ }^{38}$ ), recurrent appendicitis defined as clear clinical suspicion of acute appendicitis evaluated at follow-up after 10 days up to 1 year, late recurrence of acute appendicitis after 1 year, duration of hospital stay, VAS scores, length of sick leave, treatment costs and quality of life (QOL using either 5D or 15D validated QOL questionnaire).

\section{Data collection}

Data collection from all patients presenting with suspected acute appendicitis in the emergency room includes: tympanic temperature, nausea and its duration, pain in the right lower quadrant of the abdomen and its duration, pain migration, presence of tenderness in the right lower quadrant and the VAS score. Additionally, CT scan results and laboratory test results will be recorded. The prospectively collected data in the emergency room allows for retrospective assessment of various appendicitis scoring systems including clinical and radiological parameters. This information will be evaluated retrospectively and used to indicate that the patients recruited to both APPAC II and III trials are similar in terms of demographics and appendicitis severity. Daily follow-up data of study patients with acute appendicitis during hospitalisation will include status findings, VAS score, tympanic temperature as well as white cell count and CRP measurements. At discharge, the length of hospital stay, VAS score, profession, length of sick leave and possible prescribed analgesics are recorded. If the patient undergoes appendectomy, operative details including operation duration, approach and operative findings will be recorded. Possible wound infection and associated postoperative antibiotic therapy will be evaluated and recorded on discharge.

The follow-up after discharge for patients in the APPAC III trial will include laboratory tests (leucocyte count, CRP) at 2-4 and/or 10 days after discharge and also a phone call at 2 to 4 days depending on the day of their discharge from the hospital. All of the patients will also be evaluated at 10 days after the discharge by phone call. The follow-up will include a contact by phone at 2 months, and at $1,3,5$ and 10 years. Data collected during the follow-up include possible side effects of the treatment given including also possible appendectomy, the possible recurrence of appendicitis and the timing of the recurrence with operative findings and histopathology of the removed appendix, VAS score at control time point, additional sick leave and evaluation of abdominal symptoms. The long-term follow-up with phone interviews allows us to monitor the patients regarding possible abdominal symptoms and patients are referred to further examinations according to standard care. The patients are also instructed to contact the researchers in case of abdominal symptoms mainly in the lower-right quadrant between the standard follow-up time points. The risk of missed appendiceal malignancy is very low especially in uncomplicated acute appendicitis and the inclusion criterion of CT scan diagnosis of uncomplicated acute appendicitis adds to patient safety in this respect. 


\section{Statistical hypothesis}

The primary objective of the study is to compare the success of treatment between antibiotic therapy and placebo group within 10 days after initiation of the randomised treatment. Superiority of antibiotic group versus placebo group will be tested using following statistical hypothesis:

$\mathrm{H}_{0}: \mathrm{p}_{1} \leq \mathrm{p}_{2}$

$\mathrm{H}_{1}: \mathrm{p}_{1}>\mathrm{p}_{2}$

where $p_{1}$ is success of treatment proportion of antibiotic group and $\mathrm{p}_{2}$ for placebo group.

\section{Safety monitoring and interim analysis}

For the safety of the patients in the placebo treatment group, an interim analysis will be conducted after 46 patients are enrolled to study and followed for 10 days. The interim analysis will be performed only once during the trial regardless of the three different scenarios and sample size estimates as the selection of the scenarios is based only on the number of enrolled patients. For the interim analyses, a safety statistician with independent trial safety monitoring committee will open the treatment code and calculate the point estimate of the proportion in each group. If the proportion in at least one of the groups is below $50 \%$, the study will be terminated. The safety statistician and the independent committee members will be the only ones who know the exact proportions. The investigators and the study statistician will only be informed of the continuation or the termination of the study. No statistical tests will be conducted in interim analysis and therefore no corrections to the p-values are needed in the final analyses of study. In addition to the interim analysis, the safety monitoring committee may be asked to meet ad hoc, if evaluated necessary by the researchers. In the case of a participating patient progressing to severe sepsis or a mortality, the study will be stopped and the independent trial safety monitoring committee will review the case and unblind the treatment assignment to determine if it occurred in a patient not receiving antibiotics. The committee will then consider relatedness to the study and if the study should be permanently terminated.

\section{Statistical analyses}

Categorical variables of the study will be characterised by treatment using frequencies and percentages and for continuous variables means and SD or medians with range and 25th and 75th percentiles will be used. Difference in treatment success between antibiotic and placebo group will be tested using Fisher's one-sided test. The two-sided $90 \%$ CI for proportion difference will be calculated as well to estimate the treatment difference. The secondary outcomes will be analysed using $\chi^{2}$ test, independent samples t-test or Mann-Whitney U test. The assumptions of tests will be checked for justification of the analyses. For the secondary outcomes, two-sided p-values will be used. The study site differences will be evaluated in statistical models and if major differences are detected more complicated statistical models will be used in the analyses of primary and secondary outcomes. P-values less than 0.05 will be considered statistically significant. The main analyses will be based on the intention-to-treat (ITT) principle (all randomised excluding possible erroneously randomised such as patients with CT diagnosis of complicated appendicitis). The subjects with missing data will automatically be excluded from the analyses of the variables in concern. Statistical analyses will be performed using SAS System for Windows, V.9.4 or later.

\section{PATIENTS AND PUBLIC INVOLVEMENT}

The development of the research question and outcome measures were based on the results of our original APPAC trial $^{8}$ focusing on the patient-centred outcome of future patients gaining major benefit from the possibility of avoiding unnecessary antibiotics and unnecessary hospitalisation. In addition to the patient benefit, the research question and endpoints could have a similar impact regarding both hospital resource use of decreasing hospitalisation days and operative theatre resources and also major cost savings in a wider economic societal aspect. Patients were not directly involved in the design of this study and the burden of the intervention was not assessed by patients themselves. On recruitment, patients are thoroughly informed of the current knowledge regarding antibiotics in the treatment of CT scan-confirmed uncomplicated acute appendicitis including treatment success, possible late recurrence and safety in order to help patients make an informed decision about trial participation. Additionally, we did conduct a pilot phase study with five patients prior to actual study initiation to ensure smooth enrolment, randomisation and hospital pharmacy study medication process. After completion of data collection and data check after primary endpoint evaluation time point, the randomisation code will be accessed and all patients will be informed of their treatment arm and the study results by mail. The patients will be provided with an opportunity to ask further questions.

\section{ETHICS AND DISSEMINATION \\ Data collection and confidentiality}

Researchers have created the online database together with BCB Medical (Turku, Finland), where all patients evaluated for acute appendicitis and study enrolment will be recorded after signed informed consent is obtained. All data are handled confidentially and the information in the datasets in the analyses is non-identifiable. Data are gathered during emergency room visit, hospitalisation for acute appendicitis, clinical observations and follow-up phone calls. The information recorded from the non-participating patients is used as register-based study data. The primary investigator will be in charge of the common data base with full access to the data and otherwise the access to the data is limited. The researchers need full access to the data in order to be able to correct possible false data entries, to enter missing data and to be able to 
keep up with the number of enrolled patients. The online database will not be used for other purposes during the trial and all of the visits to the database will be recorded in the database log.

\section{Ethics}

This protocol has been accepted by the Ethical Committee of the Hospital District of Southwest Finland at Turku University Hospital and Finnish Medicines Agency (FIMEA). The trial will be conducted in compliance with the Declaration of Helsinki.

\section{Withdrawal}

Patients are informed of their right to withdraw from the study without explanation at any time. In case of patient withdrawal, they will be asked for permission for prospective collection and later use of their hospital record data after their withdrawal.

\section{Dissemination plan}

The results of this trial will be disseminated by publication in international peer-reviewed scientific journals and by presentations at international and/or domestic conferences. If the trial results warrant changes in the standard treatment of uncomplicated acute appendicitis, the widespread execution of the trial throughout Finland will assist in its implementation.

\section{DISCUSSION}

The treatment of uncomplicated acute appendicitis is under active research with non-operative management proving safe ${ }^{814-1639}$ and cost-efficient. ${ }^{32}$ Uncomplicated acute appendicitis is quite similar to uncomplicated acute diverticulitis, where recent studies have demonstrated no benefit of antibiotics compared with symptomatic treatment alone. ${ }^{17-22}$ Similar spontaneous resolution with symptomatic treatment has also been indicated in uncomplicated acute appendicitis ${ }^{23}$ and the time has come to a double-blinded evaluation of the role of antibiotics and spontaneous resolution in the treatment of uncomplicated acute appendicitis. To our knowledge, the APPAC III trial is the first study to evaluate spontaneous resolution of uncomplicated acute appendicitis in a double-blinded RCT. The depiction of spontaneous resolution is of vital clinical importance in evaluating the optimal treatment for uncomplicated acute appendicitis. If antibiotic therapy does not provide any benefit over placebo in uncomplicated acute appendicitis, this could have a profound impact on the treatment of this very common surgical emergency, especially in this era of ever-increasing antimicrobial resistance.

\section{Strengths and limitations of the study}

This trial only includes patients with CT-confirmed uncomplicated acute appendicitis, thus reducing the risk of including patients with complicated acute appendicitis, other diagnoses or a non-inflamed appendix. When selecting patients for possible non-operative treatment with either antibiotics or even placebo, the accurate differential diagnosis between uncomplicated and complicated forms of acute appendicitis is of vital clinical importance and diagnostic imaging with a high sensitivity in detecting patients with complicated acute appendicitis is needed. A recent meta-analysis ${ }^{40}$ stated 10 specific CT imaging features most informative for complicated appendicitis and all these CT findings are used as criteria for complicated appendicitis in the APPAC III study. This gold standard CT diagnostics enhances the chance of successful non-operative management. We feel that the use of CT imaging as a means to obtain accurate radiological diagnosis of uncomplicated acute appendicitis is of vital importance especially in this experimental placebo setting, but also in current clinical practice especially regarding the low-dose CT possibilities. Risk stratification scores, although useful in many settings, are not as comparable and accurate in diagnosing acute appendicitis. Additionally, the scoring systems are generally not capable in differential diagnosis between uncomplicated and complicated acute appendicitis, which is crucial in assessing the possibility of non-operative treatment.

The used CT protocols (low dose or standard) are thoroughly recorded and the radiologist use a standardised CT scan report sheet (box 1) in order to facilitate standardisation of the diagnostic imaging, which has been shown to result in high reproducibility of objective CT findings achieving high diagnostic accuracy in an at-risk population. ${ }^{41}$ This standardised recording enables thorough assessment of the accuracy of diagnostic CT imaging (low dose and/vs standard) used in this study, as well as the retrospective analysis of the accuracy of the existing scoring systems with clinical and laboratory findings used for diagnosing acute appendicitis. In our APPAC study, cost-effectiveness results comparing antibiotics or appendectomy, the imaging costs were a minor portion of the total costs of either treatment ${ }^{32}$ and the use of CT scan has been shown to markedly reduce the treatment costs by avoiding unnecessary negative appendectomies.

To further guarantee patient safety, this protocol dictates systematic and frequent evaluation of the trial patients by clinical assessment and laboratory tests increasing the likelihood of early identification of the patients not responding to the double-blinded treatment. The patients are closely monitored at the hospital for the whole 3-day duration of the intravenous treatment by the research group senior surgeons, who decide on possible approach of direct appendectomy in cases of suspected non-responsiveness further improving patient safety. A follow-up phone call is scheduled for 2 to 4 days and 10 days after discharge to ensure patient safety and evaluation of treatment success. Additionally, predefined interim analysis by an outside safety monitoring committee will be performed further assuring patient safety.

In addition to the undisputed advantages in differential diagnostics between uncomplicated and complicated acute appendicitis, routine CT imaging in the diagnosis of acute appendicitis is also known to reduce the rate 
of negative appendectomies, ${ }^{42}$ surgical complications and treatment costs. ${ }^{31}$ There is accumulating evidence showing that low-dose CT protocols are as accurate as the standard protocols in diagnosing acute appendicitis, ${ }^{27} 34$ thereby offering means to reduce the amount of ionising radiation resulting from the diagnostic CT imaging of acute appendicitis. However, we decided to use standard protocol for obese patients in the APPAC III trial, since the OPTICAP phantom trial ${ }^{35}$ conducted by the study group endorsed the view shared by Poletti $e t a t^{43}$ that diagnostic accuracy of low-dose abdominal CT protocols may not yet be adequate in patients with BMI exceeding $30 \mathrm{~kg} / \mathrm{m}^{2}$.

The double-blinded setting is extremely essential in excluding potential bias on behalf of both the patient and the treating surgeon in order to provide reliable evidence against or for spontaneous resolution of uncomplicated acute appendicitis. However, double-blinded surgical trials are challenging to conduct even in an elective setting and even more so in the emergency setting especially with the major limitation of eligible enrolment time frame dependent on the hospital pharmacy working hours. In addition, according to the study ethics approval, to ensure patient safety, all patients are recruited by senior research surgeons and not the surgeon on call further adding to time schedule challenges based on researcher availability. Thirdly, the working hours of the hospital pharmacy are not in concurrence with the admission hours of patients with suspected acute appendicitis. The maintenance of hospital pharmacy resources is also a challenge to trial funding as manufacturing and delivering the study drugs is quite expensive. Based on these aforementioned challenges in this trial, we anticipate possible delays in enrolment and thus have created three scenarios for patient enrolment number and study power analysis. We acknowledge these alternate scenarios and sample size calculation to represent a limitation in this study, but the acknowledgement of these true challenges and preparing for alternate scenarios based on enrolment speed assures the completion of this trial within reasonable time schedule in a real-life emergency surgery setting. Even in the slowest enrolment scenario C with quite a high minimal clinically important difference, the treatment success rate of placebo may end up representing quite a significant treatment success rate with placebo even though it would not illustrate superiority of antibiotic therapy. However, despite this acknowledged limitation of scenario $\mathrm{C}$, it could still confirm the existence of spontaneous resolution of uncomplicated acute appendicitis in the majority of patients in a doubleblinded study. This would markedly add to current knowledge and would provide an excellent basis for large open-label future trials evaluating symptomatic treatment in uncomplicated acute appendicitis.

The concurrent enrolment to two different trials (APPAC III and APPAC II) could be evaluated as a limitation potentially being a source of selection bias. However, this potential risk of bias is very minor as the only difference between enrolments in the two trials is time of day (depending on hospital pharmacy working hours) and patients are recruited to both APPAC II and III trials based on identical inclusion and exclusion criteria. Based on these identical inclusion criteria, it would be highly unlikely that less severe cases will be enrolled into this APPAC III trial. Further supporting this lack of bias is the fact that APPAC III recruitment will be performed by the senior research surgeons of the APPAC study group with vast clinical experience and awareness of the trial protocol compared with surgeons on call mostly having much less clinical experience.

The 3-day required hospitalisation in this protocol can be considered a limitation of the study as it has been shown that early hospital discharge following antibiotic treatment for uncomplicated acute appendicitis promotes return to normal function. ${ }^{44}$ However, in this experimental trial setting, the 3-day hospitalisation was evaluated necessary in order to ensure patient safety. In addition, the duration of the antibiotic treatment is 7 days even though recent data show that after adequate source control outcomes after short duration antibiotics for intra-abdominal infections are similar to those after a longer course of antibiotics. ${ }^{45}$ As there is no source control in antibiotic or symptomatic treatment of uncomplicated acute appendicitis, the duration of the antibiotic therapy was set at 1 week. The choice of antibiotic regimen was based on our previous APPAC trial, but the duration of the antibiotic treatment was already shortened from the original 10 days to 7 days in this APPAC III trial.

\section{Choice of primary outcome and antibiotic regimen}

Determining the primary outcome in both study groups is very similar as the treatment interventions are very similar opposed to our previous APPAC trial ${ }^{8}$ comparing appendectomy with antibiotic treatment. Spontaneous resolution has already been shown in uncomplicated acute diverticulitis ${ }^{17-22}$ and recently also in one single-blinded study comparing antibiotic therapy and no antibiotic therapy for acute appendicitis published after planning of this double-blinded APPAC III trial, ${ }^{23}$ but to our knowledge, no double-blinded study has been conducted so far. The antibiotic regimen used in this study has already been deemed safe and efficient in the APPAC study. ${ }^{8}$

\section{Effects on gut microbiota and antibiotic resistance}

Antimicrobial resistances (AMR) is considered an increasing problem in healthcare. The prudent use of antimicrobials is essential to prevent increasing AMR. Additionally, antimicrobials are known to decrease the diversity of the gut microbiota, richness and species variation and cause perturbation of its overall balance and even a short-term antimicrobial treatment has a longterm impact on the gut microbiota composition underlining the importance of evaluating both short- term and long-term effects of antimicrobial treatment in old and new indications. ${ }^{47}$ Symptomatic treatment of uncomplicated acute appendicitis also carries the advantage of 
avoiding the adverse events of antibiotics ranging from typical mild gastrointestinal symptoms to rare cases of antibiotic-related colitis. In the pilot study by Talan et $a l$, antibiotic-treated patients experienced less pain and disability but twice as many mostly mild symptoms of diarrhoea, headache and nausea compared with the surgery group. ${ }^{44}$ Thus, it is sensible to explore the role of symptomatic treatment without antibiotics instead of antimicrobial treatment in uncomplicated acute appendicitis in a controlled setting ensuring patient safety. Alternatively, antibiotics are generally safe and may be potentially lifesaving. Treatment targeted only to patients with CT-confirmed uncomplicated acute appendicitis and a limited treatment duration of 7 days is unlikely to be of great relative importance in the promotion of community bacterial resistance.

\section{Patients unwilling to participate}

Although recent scientific evidence proves non-operative treatment for uncomplicated acute appendicitis safe, the general understanding among most people still is that an acute appendicitis needs to be operated. This might result in patients unwilling to participate in the study. In order to understand selection bias, we designed the study protocol to include thorough recording of data on all patients evaluated for eligibility.

\section{CONCLUSION}

The APPAC III trial is a randomised controlled doubleblinded multicentre study comparing antibiotic therapy with placebo in the treatment of uncomplicated acute appendicitis.

\author{
Author affiliations \\ ${ }^{1}$ Division of Digestive Surgery and Urology, Turku University Hospital, Turku, Finland \\ ${ }^{2}$ Department of Surgery, University of Turku, Turku, Finland \\ ${ }^{3}$ Department of Surgery, Helsinki University Central Hospital, Helsinki, Finland \\ ${ }^{4}$ Department of Surgery, Oulu University Hospital, Oulu, Finland \\ ${ }^{5}$ Division of Surgery, Gastroenterology and Oncology, Tampere University Hospital, \\ Tampere, Finland \\ ${ }^{6}$ Department of Surgery, Kuopio University Hospital, Kuopio, Finland \\ ${ }^{7}$ Department of Biostatistics, University of Turku, Turku, Finland \\ ${ }^{8}$ Department of Radiology, Turku University Hospital, Turku, Finland
}

Correction notice This article has been corrected since it was first published. The following typo $\mathrm{H} 0: \mathrm{p} 1=\mathrm{p} 2 \mathrm{H} 1: \mathrm{p} 1>\mathrm{p} 2$ has been corrected to $\mathrm{H} 0: \mathrm{p} 1 \leq \mathrm{p} 2 \mathrm{H} 1$ : p1 $>$ p2.

Acknowledgements We acknowledge all patients evaluated for study enrolment and especially all participating patients, supporting surgeons, radiologists, emergency medicine physicians and nurses and also all study hospital pharmacies contributing in carrying out this APPAC III trial.

Contributors All authors have contributed to the design of this trial protocol. PS is the chief investigator. PS, SS and JG have initiated the project. The protocol was drafted by PS, which was refined by SS, JG, VS, TR, PN, TR, AL, SM, JL, HS, JV and SH. Statistical advice was provided by SH. SS was responsible for drafting the protocol manuscript, which was refined by PS. All authors have read and approved the final manuscript. The APPAC collaborative study group consists of all local investigators who are responsible for execution of the trial and valid data gathering.

Funding This work was supported by the Finnish Society of Digestive Surgeons, which covered the costs of the online database created together with the researchers and BCB Medical. This work was also supported by a government research grant awarded to Turku University Hospital (EVO Foundation) for PS and SS. Additional support from three research grants awarded to both SS (Orion Pharma research foundation and the Finnish Gastroenterological Research Foundation) and PS (Sigrid Juselius Foundation).

Competing interests PS has received lecture fees from Merck, Lilly and Orion Pharma.

Ethics approval The trial has been approved by the Ethics Committee of the Hospital District of Southwest Finland and the Finnish Medicines Agency FIMEA.

Provenance and peer review Not commissioned; externally peer reviewed.

Open access This is an open access article distributed in accordance with the Creative Commons Attribution Non Commercial (CC BY-NC 4.0) license, which permits others to distribute, remix, adapt, build upon this work non-commercially, and license their derivative works on different terms, provided the original work is properly cited, appropriate credit is given, any changes made indicated, and the use is non-commercial. See: http://creativecommons.org/licenses/by-nc/4.0/.

\section{REFERENCES}

1. McBurney C. Experience with early operative interference in cases of the vermiform appendix. NY Med J 1889;50:676-84.

2. Livingston EH, Fomby TB, Woodward WA, et al. Epidemiological similarities between appendicitis and diverticulitis suggesting a common underlying pathogenesis. Arch Surg 2011;146:308-14.

3. Leung TT, Dixon E, Gill M, et al. Bowel obstruction following appendectomy: what is the true incidence? Ann Surg 2009;250:51-3.

4. Margenthaler JA, Longo WE, Virgo KS, et al. Risk factors for adverse outcomes after the surgical treatment of appendicitis in adults. Ann Surg 2003;238:59-66.

5. Dai L, Shuai J. Laparoscopic versus open appendectomy in adults and children: a meta-analysis of randomized controlled trials. United European Gastroenterol J 2017;5:542-53.

6. Fitz R. Perforating inflammation of the vermiform appendix. Am J Med Sci 1886;92:321-46.

7. McBurney C. II. The indications for early laparotomy in Appendicitis. Ann Surg 1891;13:233-54.

8. Salminen P, Paajanen H, Rautio T, et al. Antibiotic therapy vs appendectomy for treatment of uncomplicated acute appendicitis: the APPAC Randomized Clinical Trial. JAMA 2015;313:2340-8.

9. Vons C, Barry C, Maitre S, et al. Amoxicillin plus clavulanic acid versus appendicectomy for treatment of acute uncomplicated appendicitis: an open-label, non-inferiority, randomised controlled trial. Lancet 2011;377:1573-9.

10. Hansson J, Körner U, Ludwigs K, et al. Antibiotics as first-line therapy for acute appendicitis: evidence for a change in clinical practice. World J Surg 2012;36:2028-36.

11. Styrud J, Eriksson S, Nilsson I, et al. Appendectomy versus antibiotic treatment in acute appendicitis. a prospective multicenter randomized controlled trial. World J Surg 2006;30:1033-7.

12. Varadhan KK, Humes DJ, Neal KR, et al. Antibiotic therapy versus appendectomy for acute appendicitis: a meta-analysis. World J Surg 2010;34:199-209.

13. Sallinen V, Tikkinen KA. Antibiotics or Appendectomy for acute non-perforated appendicitis--how to interpret the evidence? Scand J Surg 2016;105:3-4.

14. Harnoss JC, Probst $P$, Büchler MW, et al. Antibiotics versus appendicectomy for the treatment of uncomplicated acute appendicitis: an updated meta-analysis of Randomised Controlled Trials by Rollins et al. World J Surg 2017;41:2411.

15. Sallinen V, Akl EA, You JJ, et al. Meta-analysis of antibiotics versus appendicectomy for non-perforated acute appendicitis. $\mathrm{Br} J$ Surg 2016;103:656-67.

16. Sakran JV, Mylonas KS, Gryparis A, et al. Operation versus antibiotics-the appendicitis conundrum continues: a meta-analysis. J Trauma Acute Care Surg 2017;82:1129-37.

17. Chabok A, Påhlman L, Hjern F, et al. Randomized clinical trial of antibiotics in acute uncomplicated diverticulitis. Br J Surg 2012;99:532-9.

18. Daniels L, Ünlü Ç, de Korte N, et al. Randomized clinical trial of observational versus antibiotic treatment for a first episode of CTproven uncomplicated acute diverticulitis. Br J Surg 2017;104:52-61.

19. Isacson $\mathrm{D}$, Andreasson $\mathrm{K}$, Nikberg M, et al. No antibiotics in acute uncomplicated diverticulitis: does it work? Scand J Gastroenterol 2014;49:1441-6.

20. de Korte N, Kuyvenhoven JP, van der Peet DL, et al. Mild colonic diverticulitis can be treated without antibiotics. A case-control study. Colorectal Dis 2012;14:325-30. 
21. Isacson D, Thorisson A, Andreasson K, et al. Outpatient, nonantibiotic management in acute uncomplicated diverticulitis: a prospective study. Int J Colorectal Dis 2015;30:1229-34.

22. Mali JP, Mentula PJ, Leppäniemi AK, et al. Symptomatic treatment for uncomplicated acute diverticulitis: a prospective cohort study. Dis Colon Rectum 2016;59:529-34

23. Park HC, Kim MJ, Lee BH. Randomized clinical trial of antibiotic therapy for uncomplicated appendicitis. Br J Surg 2017;104:1785-90.

24. Körner H, Söndenaa K, Söreide JA, et al. Incidence of acute nonperforated and perforated appendicitis: age-specific and sexspecific analysis. World J Surg 1997;21:313-7.

25. Lietzén E, Mällinen J, Grönroos JM, et al. Is preoperative distinction between complicated and uncomplicated acute appendicitis feasible without imaging? Surgery 2016:160:789-95.

26. Atema JJ, van Rossem CC, Leeuwenburgh MM, et al. Scoring system to distinguish uncomplicated from complicated acute appendicitis. Br J Surg 2015;102:979-90.

27. Kim HJ, Jeon BG, Hong CK, et al. Low-dose CT for the diagnosis of appendicitis in adolescents and young adults (LOCAT): a pragmatic, multicentre, randomised controlled non-inferiority trial. Lancet Gastroenterol Hepatol 2017;2:793-804.

28. Rao PM, Rhea JT, Novelline RA, et al. Effect of computed tomography of the appendix on treatment of patients and use of hospital resources. N Engl J Med 1998;338:141-6.

29. Paulson EK, Kalady MF, Pappas TN. Clinical practice. Suspected appendicitis. N Engl J Med 2003;348:236-42.

30. Boonstra PA, van Veen RN, Stockmann HB. Less negative appendectomies due to imaging in patients with suspected appendicitis. Surg Endosc 2015;29:2365-70.

31. Lahaye MJ, Lambregts DM, Mutsaers E, et al. Mandatory imaging cuts costs and reduces the rate of unnecessary surgeries in the diagnostic work-up of patients suspected of having appendicitis. Eur Radiol 2015;25:1464-70.

32. Sippola S, Grönroos J, Tuominen R, et al. Economic evaluation of antibiotic therapy versus appendicectomy for the treatment of uncomplicated acute appendicitis from the APPAC randomized clinical trial. Br J Surg 2017;104:1355-61.

33. Kim K, Kim YH, Kim SY, et al. Low-dose abdominal CT for evaluating suspected appendicitis. N Engl J Med 2012;366:1596-605.

34. Yun SJ, Ryu CW, Choi NY, et al. Comparison of low- and standarddose CT for the diagnosis of acute appendicitis: a meta-analysis. AJR Am J Roentgenol 2017;208:W198-W207.
35. Niiniviita H, Salminen P, Grönroos JM, et al. Low-dose ct protocol optimization for the assessment of acute appendicitis: the opticap phantom study. Radiat Prot Dosimetry 2018;178:20-8.

36. Sippola S, Virtanen J, Tammilehto V, et al. The Accuracy of Low-dose Computed Tomography Protocol in Patients With Suspected Acute Appendicitis: The OPTICAP Study. Ann Surg 2018:1. doi: 10.1097/ SLA.0000000000002976. [Epub ahead of print].

37. Chan AW, Tetzlaff JM, Altman DG, et al. SPIRIT 2013 statement: defining standard protocol items for clinical trials. Ann Intern Med 2013;158:200-7.

38. Clavien PA, Barkun J, de Oliveira ML, et al. The Clavien-Dindo classification of surgical complications: five-year experience. Ann Surg 2009;250:187-96.

39. Rollins KE, Varadhan KK, Neal KR, et al. Antibiotics versus appendicectomy for the treatment of uncomplicated acute appendicitis: an updated meta-analysis of randomised controlled trials. World J Surg 2016;40:2305-18.

40. Kim HY, Park JH, Lee YJ, et al. Systematic review and meta-analysis of CT Features for differentiating complicated and uncomplicated appendicitis. Radiology 2018;287.

41. Simianu VV, Shamitoff A, Hippe DS, et al. The reliability of a standardized reporting system for the diagnosis of appendicitis. Curr Probl Diagn Radiol 2017;46:267-74

42. van Rossem CC, Bolmers MD, Schreinemacher MH, et al. Diagnosing acute appendicitis: surgery or imaging? Colorectal Dis 2016;18:1129-32.

43. Poletti PA, Becker M, Becker CD, et al. Emergency assessment of patients with acute abdominal pain using low-dose CT with iterative reconstruction: a comparative study. Eur Radiol 2017;27:3300-9.

44. Talan DA, Saltzman DJ, Mower WR, et al. Antibiotics-First versus surgery for appendicitis: a US Pilot randomized controlled trial allowing outpatient antibiotic management. Ann Emerg Med 2017;70:1-11.

45. Celestin AR, Odom SR, Angelidou K, et al. Novel method suggests global superiority of short-duration antibiotics for intra-abdominal infections. Clin Infect Dis 2017;65:1577-9.

46. Sawyer RG, Claridge JA, Nathens AB, et al. Trial of short-course antimicrobial therapy for intraabdominal infection. $N$ Engl J Med 2015;372:1996-2005.

47. Lange K, Buerger M, Stallmach A, et al. Effects of Antibiotics on Gut Microbiota. Dig Dis 2016;34:260-8. 